\title{
Periodic structure in nuclear matter
}

\author{
István Lovas and Lívia Molnár \\ Department of Theoretical Physics, Lajos Kossuth University, H-4010 Debrecen, Hungary \\ Kornél Sailer* and Walter Greiner \\ Institut für Theoretische Physik, Johann Wolfgang Goethe Universität, Frankfurt am Main, Federal Republic of Germany
}

(Received 8 July 1991)

\begin{abstract}
The properties of nuclear matter are studied in the framework of quantum hadrodynamics. Assuming an $\omega$-meson field, periodic in space, a self-consistent set of equations is derived in the mean-field approximation for the description of nucleons interacting via $\sigma$-meson and $\omega$-meson fields. Solutions of these self-consistent equations have been found: The baryon density is constant in space, however, the baryon current density is periodic. This high density phase of nuclear matter can be produced by anisotropic external pressure, occurring, e.g., in relativistic heavy ion reactions. The self-consistent fields developing beyond the instability limit have a special screw symmetry. In the presence of such an $\omega$ field, the energy spectrum of the relativistic nucleons exhibits allowed and forbidden bands, similar to the energy spectrum of the electrons in solids.
\end{abstract}

PACS number(s): 21.65. $+\mathrm{f}$

\section{INTRODUCTION}

In a great number of physical systems, the homogeneous, isotropic distribution of matter looses its stability and at some specific values of the physical parameters a periodic structure is formed spontaneously [1]. In nuclear physics the pion condensation [2] may serve as a typical example for such a phenomenon. In the last few years, the instabilities of homogeneous nuclear matter leading to the formation of a periodic meson field have been discussed in a number of papers [3-6].

The possibility of a periodic structure associated with a periodic $\omega$ field is expected for the following reason. The periodic structure corresponds to a specific combination of particle-hole excitations of the nucleons, which requires some excitation energy. The $\omega$ field, which mediates a repulsive interaction in the particle-particle channel, gives rise to an attraction in the particle-hole channel. If the energy gain due to this attractive particle-hole interaction overcompensates the energy loss needed to excite the particle-hole configuration, the formation of a periodic structure is favored. A similar phenomenon can be observed in electron plasma [7,8].

In this paper, working in the framework of quantum hadrodynamics (QHD) [9], a periodic $\omega$ field is assumed and a set of self-consistent equations is derived in the relativistic mean-field approximation. In this way the periodic structure can be studied also beyond the instability point of the homogeneous system.

It has to be noted that, for the moment, the reliability of the mean-field approximation is not yet clarified.

Studying the possibility of periodic structures, Friman and Henning [4] have investigated the condition of the in-

\footnotetext{
*Present address: Department of Theoretical Physics, Lajos Kossuth University, H-4010 Debrecen, Hungary.
}

stability. They have found the limits of the instability in the mean-field approximation. The instability, however, disappears when the quantum corrections in the one-loop approximation are included. From this observation they have arrived at the conclusion that the instability against the formation of a periodic structure found in the meanfield approximation is a spurious, nonphysical effect. At the same time, another type of instability has been found by Furnstahl and Horowitz [5] calculating the meson propagators at the one-loop level. Using the randomphase approximation, Price, Shepard, and McNeil have shown the possibility of a periodic structure which can be associated with alpha-particle clustering [6]. The problem seems to be even more involved if we take into account the results of Ref. [10] where it was proved that the perturbative corrections to the mean-field approximation in terms of one-loop, two-loop, etc., contributions do not form a convergent series. From these studies it follows that the results obtained in the mean-field approximation must be checked and corrected by nonperturbative methods [11], e.g., by lattice QHD calculations [12].

\section{MODEL}

The field equations for the nucleon field $\psi(x)$ and for the $\sigma$ - and $\omega$-meson fields are given in the standard notation as follows:

$$
\begin{aligned}
& \left\{\gamma_{\mu}\left[i \partial^{\mu}-g_{\omega} \omega^{\mu}(x)\right]+g_{\sigma} \sigma(x)-m\right\} \psi(x)=0, \\
& \square \sigma+m_{\sigma}^{2} \sigma=g_{\sigma} \bar{\psi} \psi-\frac{d U}{d \sigma}, \\
& \square \omega_{\mu}+m_{\omega}^{2} \omega_{\mu}-\partial_{\mu}\left(\partial^{v} \omega_{v}\right)=g_{\omega} \bar{\psi} \gamma_{\mu} \psi .
\end{aligned}
$$

For the sake of the flexibility of the model here, we have introduced a self-interacting $\sigma$ field with the potential $U(\sigma)$ given by

$$
U(\sigma)=\left(\frac{1}{3} b m+\frac{1}{4} c g_{\sigma} \sigma\right)\left(g_{\sigma} \sigma\right)^{3} .
$$


The parameters of this potential $(b, c)$ together with the masses $\left(m, m_{\sigma}, m_{\omega}\right)$ and coupling constants $\left(g_{\sigma}, g_{\omega}\right)$ are given in Table $\mathrm{I}$.

In the spirit of the mean-field approximation, we assume that the meson-field operators can be replaced by their expectation values considered as classical fields. The density operators of the nucleon field, i.e., the source terms of the meson fields in Eqs. (2) and (3), are also replaced by their expectation values. More specifically, we introduce the following Ansätze:

$$
\begin{aligned}
& \sigma(x) \rightarrow\langle\sigma(x)\rangle=\bar{\sigma}, \\
& \omega^{0}(x) \rightarrow\left\langle\omega^{0}(x)\right\rangle=\bar{\omega}^{0}, \\
& \omega^{1}(x) \rightarrow\left\langle\omega^{1}(x)\right\rangle=\bar{\omega} \cos k z, \\
& \omega^{2}(x) \rightarrow\left\langle\omega^{2}(x)\right\rangle=-\bar{\omega} \sin k z, \\
& \omega^{3}(x) \rightarrow\left\langle\omega^{3}(x)\right\rangle=0, \\
& \bar{\psi} \Gamma \psi \rightarrow\langle\bar{\psi} \Gamma \psi\rangle .
\end{aligned}
$$

Here $z$ denotes the $x^{3}$ component of the $x$ four-vector and $k\left(\equiv k^{3}\right)$ is the only nonvanishing component of the wave-number four-vector for the $\omega$ field.

By substituting these Ansätze into the field equations, we arrive at the following set of equations:

$$
\begin{aligned}
& {\left[i \gamma_{\mu} \partial^{\mu}-g_{\omega} \bar{\omega}^{0} \gamma^{0}\right.} \\
& \left.\quad+g_{\omega} \bar{\omega}\left(\gamma^{1} \cos k z-\gamma^{2} \sin k z\right)-m+g_{\sigma} \bar{\sigma}\right] \psi(x)=0, \\
& m_{\sigma}^{2} \bar{\sigma}=g_{\sigma}\langle\bar{\psi} \psi\rangle-\frac{d U}{d \bar{\sigma}}, \\
& m_{\omega}^{2} \bar{\omega}^{0}=g_{\omega}\left\langle\bar{\psi} \gamma^{0} \psi\right\rangle, \\
& \left(k^{2}+m_{\omega}^{2}\right) \bar{\omega} \cos k z=g_{\omega}\left\langle\bar{\psi} \gamma^{1} \psi\right\rangle, \\
& -\left(k^{2}+m_{\omega}^{2}\right) \bar{\omega} \sin k z=g_{\omega}\left\langle\bar{\psi} \gamma^{2} \psi\right\rangle, \\
& 0=g_{\omega}\left\langle\bar{\psi} \gamma^{3} \psi\right\rangle .
\end{aligned}
$$

These equations determine the parameters of the assumed mean fields, i.e., the values of $\bar{\sigma}, \bar{\omega}^{0}$, and $\bar{\omega}$.

In the Ansätze given by Eqs. (5)-(9), we have assumed that an $\omega$ field periodic in space can be developed. It is easy to see that the $\omega$ field satisfies the following "Lorentz" condition:

$$
\partial_{\mu} \omega^{\mu}=0
$$

The field tensor, defined as

$$
F^{\mu v}=\partial^{v} \omega^{\mu}-\partial^{\mu} \omega^{v},
$$

has only two nonvanishing elements:

$$
\begin{aligned}
& F^{13}=\bar{\omega} k \sin k z \equiv H^{2}, \\
& F^{23}=\bar{\omega} k \cos k z \equiv-H^{1} .
\end{aligned}
$$

This "magnetic" field is perpendicular to the $z$ axis, and it rotates in the $x-y$ plane as one goes along the $z$ axis.

The $\omega$ field has a special symmetry. Namely, the $\omega$ field is invariant against a screw transformation which is the combination of a translation along the $z$ axis,

$$
z^{\prime}=z+a
$$

and a simultaneous rotation around the $z$ axis,

$$
\begin{aligned}
\omega^{\prime} & =\mathbf{B} \omega, \\
\mathbf{B} & =\left[\begin{array}{ccc}
\cos k a & -\sin k a & 0 \\
\sin k a & \cos k a & 0 \\
0 & 0 & 1
\end{array}\right],
\end{aligned}
$$

where the angle of rotation is proportional with the translation.

Furthermore, there exists a discrete translational symmetry due to the periodic behavior of the $\omega$ field. Consequently, the solutions of the Dirac equation are Bloch waves:

$$
\Psi_{i}(p, x)=e^{-i p x} \Phi_{i}(\mathbf{p}, z),
$$

where the function $\Phi_{i}$ must be periodic with the "lattice spacing" $2 \pi / k$ :

$$
\Phi_{i}(\mathrm{p}, z)=\Phi_{i}(\mathrm{p}, z+2 \pi / k) .
$$

The quasinucleon states described by Eqs. (22) and (23) are plane waves modulated by the periodic $\omega$ field. At a fixed value of the momentum $\mathbf{p}$, the function $\Phi_{i}(\mathbf{p}, z)$ is written in the form of a Fourier series:

$$
\Phi_{i}(\mathbf{p}, z)=\lim _{N \rightarrow \infty} \sum_{n=-N}^{N} u_{i}(\mathbf{p}, n) e^{-i n k z} .
$$

The bispinor $u_{i}(\mathbf{p}, n)$ together with the energy eigenvalues $E_{i}(\mathbf{p})\left(\equiv p_{i}^{0}\right)$ can be obtained by the diagonalization of the Hamiltonian

$$
\sum_{n^{\prime}} H_{n n^{\prime}} u_{i}\left(\mathbf{p}, n^{\prime}\right)=E_{i} u_{i}(\mathbf{p}, n)
$$

TABLE I. Parameters of the mesonic fields.

\begin{tabular}{lccccccc}
\hline & \multicolumn{2}{c}{$\begin{array}{c}c \\
\end{array}$} & \multicolumn{2}{c}{ parameters } & \multicolumn{2}{c}{ Coupling constants } & \multicolumn{3}{c}{ Particle masses $\left(\mathrm{fm}^{-1}\right)$} \\
& $b$ & $c$ & $g_{\sigma}$ & $g_{\omega}$ & $m$ & $m_{\sigma}$ & $m_{\omega}$ \\
\hline $\begin{array}{l}\text { "Soft" nuclear } \\
\text { matter" }\end{array}$ & -0.734 & 6.856 & 3.512 & 3.874 & & & \\
$\begin{array}{l}\text { "Stiff" nuclear } \\
\text { matter }\end{array}$ & 0.0 & 0.0 & 9.573 & 11.67 & 4.570 & 2.788 & \\
\hline \hline
\end{tabular}

${ }^{\mathrm{a}}$ Lovas, Németh, and Sailer [13].

${ }^{b}$ Walecka [9]. 

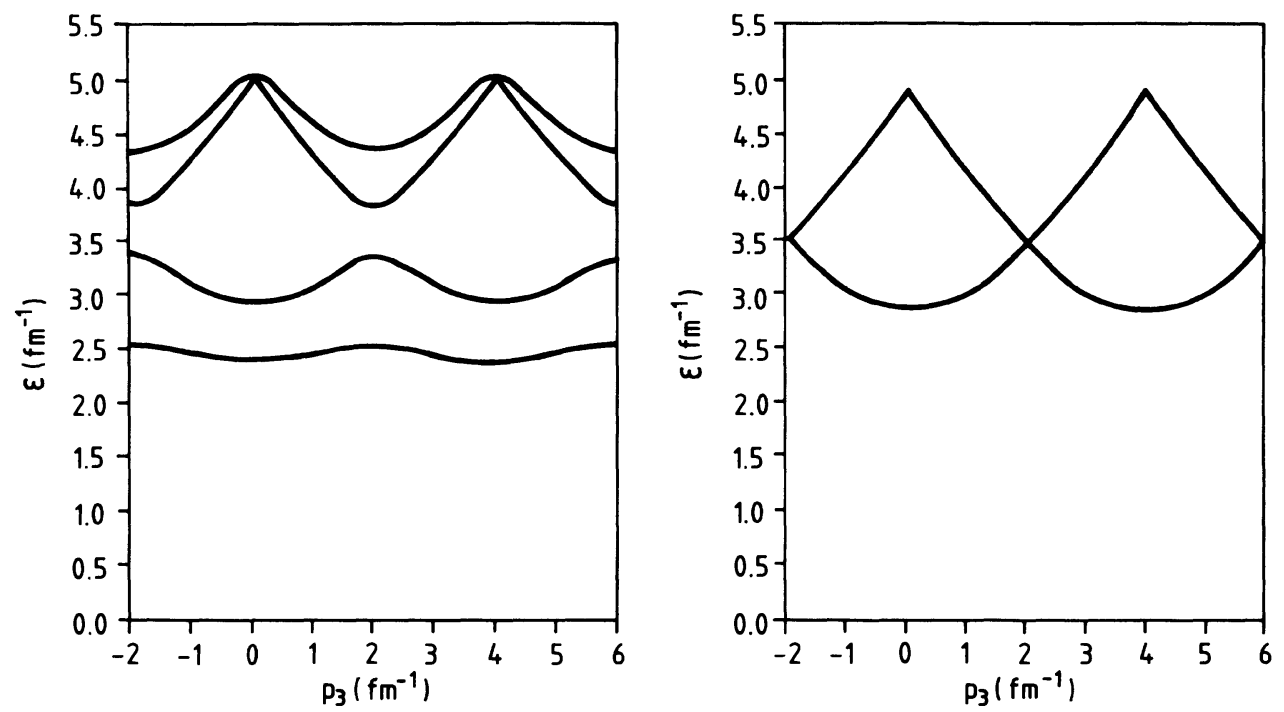

FIG. 1. Lowest positive energy levels $\varepsilon=E-g_{\omega} \bar{\omega}_{0}$ plotted against momentum component $p_{z}$ for a nucleon moving in a periodic $\omega$ field $\left(k=4.0, p_{x}=p_{y}=2.0\right)$. Left side, $\bar{\omega}=0.1$; right side, $\bar{\omega}=0.0$.

where

$$
\begin{aligned}
H_{n n^{\prime}}= & \gamma^{0}\left(\gamma \cdot \mathbf{p}+\gamma^{3} n k+m^{*}+\gamma^{0} \boldsymbol{g}_{\omega} \bar{\omega}^{0}\right) \delta_{n n^{\prime}} \\
& -\frac{1}{2} g_{\omega} \bar{\omega} \gamma^{0}\left(\gamma^{(-)} \delta_{n+1, n^{\prime}}+\gamma^{(+)} \delta_{n-1, n^{\prime}}\right)
\end{aligned}
$$

and

$$
\gamma^{( \pm)}=\left(\gamma^{1} \pm i \gamma^{2}\right)
$$

According to this set of equations, we have to diagonalize the Hamiltonian (26) in a $4(2 N+1)$-dimensional space. However, when we need only the energy eigenvalues, we can solve the equivalent eigenvalue problem given in the Appendix, which leads to a diagonalization in a $2(2 N+1)$-dimensional space.
The energy spectrum $E_{i}(\mathbf{p})$ is somewhat similar to the spectrum of crystalline solids having allowed and forbidden bands.

At every given value of $\mathbf{p}$, there exist two different states associated with the two spin degrees of freedom. These states are degenerate when the amplitude of the periodic field $\bar{\omega}$ vanishes, as can be seen on the right-hand side of Fig. 1. When the amplitude of the periodic field $\bar{\omega}$ is nonzero, the spin degeneracy is resolved. Allowed and forbidden energy bands are formed, as shown in Fig. 1. The splitting of the degeneracy can be seen in Fig. 2 when the $\bar{\omega}$-field amplitude is growing from zero to a finite value.

The expectation values of the source terms of the field equations (12)-(16) can be expressed as follows:

$$
\left\langle\bar{\psi} \gamma^{v} \psi\right\rangle=\sum_{i} \sum_{n=-N}^{+N} \sum_{n^{\prime}=-N}^{+N} e^{i\left(n^{\prime}-n\right) k z} \int_{-\infty}^{+\infty} d p_{1} \int_{-\infty}^{+\infty} d p_{2} \int_{-k / 2}^{+k / 2} d p_{3} \Theta\left(T, \mu, E_{i}(\mathbf{p})\right) \bar{u}_{i}\left(\mathbf{p}, n^{\prime}\right) \gamma^{v} u_{i}(\mathbf{p}, n)
$$

The Fermi-Dirac distribution function $\Theta$ at a fixed temperature $T$ and baryon chemical potential $\mu$ is given by

$$
\Theta\left(T, \mu, E_{i}(\mathbf{p})\right)=\left(1+e^{\left[E_{i}(\mathbf{p})-\mu\right] / T}\right)^{-1} .
$$

The integration on the first Brillouin zone from $-k / 2$ to $+k / 2$ together with the summation on the complete set of states labeled by the index $i$ is equivalent with the integration on the full momentum space in the case of nonperiodic systems.

The three-dimensional integration was performed by Gauss quadrature.

The calculations were carried out by truncating the Hilbert space at $N=3$. The results calculated at $T=20$ $\mathrm{MeV}$ are shown in Figs. 3-8.
At a few values of the parameters, we checked the rapidity of the convergence of the Fourier expansion. The calculations have been performed truncating the Hilbert space at $N=1,3$, and 5 .

The differences between the numerical values of the physical quantities calculated at $N=1$ and 3 are not negligible. However, the differences between $N=3$ and 5 are already insignificant.

We have found by direct calculation that the coefficients of $e^{-i\left(n-n^{\prime}\right) k z}$ in the source terms on the right-hand side of (12) and (13) were more than five orders of magnitude smaller in the case $n^{\prime} \neq n$ than those with $n^{\prime}=n$. At the same time, in the source terms in (14) and (15), the only nonvanishing terms are $n^{\prime}=n \pm 1$. That is, the solutions obtained with the Ansätze (5)-(9) and 


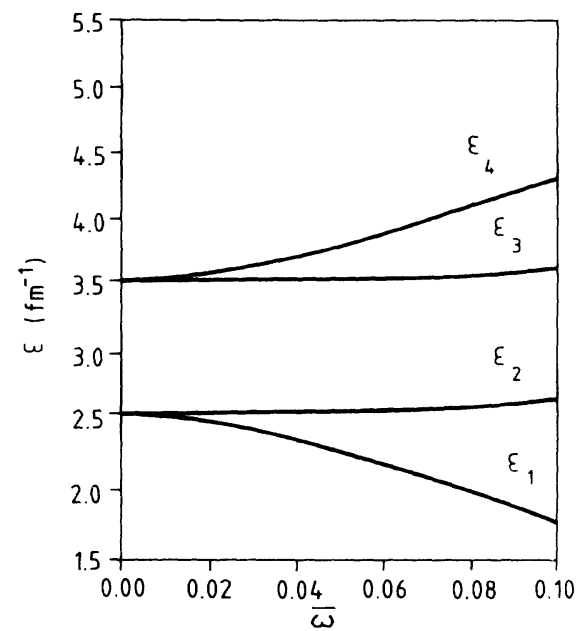

FIG. 2. Splitting of the energy levels $\varepsilon=E-g_{\omega} \bar{\omega}_{0}$ in the presence of a periodic $\omega$ field $\left(k=6.0, p_{x}=p_{y}=0.0, p_{z}=2.5\right)$ (inside the first Brillouin zone).

$(22)-(24)$ are self-consistent with a high accuracy. This means that the $e^{-i n k z}(n=1,2, \ldots, N)$ type modulations of the plane-wave states are not present in the nucleon current $\left\langle\bar{\psi} \gamma^{\mu} \psi\right\rangle$ except for the components $\mu=1$ and 2, which are modulated only by $\cos k z$ and $-\sin k z$, respectively. In other words, there are no higher-order har- monics in the nucleon current.

To facilitate the physical interpretation of the selfconsistent solution, we reformulate the whole problem in the language of statistical physics in the next section.

\section{OF THE THERMODYNAMICAL POTENTIAL}

Let us introduce the thermodynamical potential $\Omega$ of the system using the standard definition

$$
\Omega=-T \ln Z,
$$

where the grand canonical partition function $Z$ is defined as usual:

$$
Z=\operatorname{Tr} e^{-(H-\mu B) / T}
$$

where the Hamiltonian of the system and the operator of the baryon number are denoted by $H$ and $B$, respectively.

In this model it is assumed that the nucleons interact only via the mesonic mean fields; consequently, the system can be considered as a system of noninteracting quasinucleons occupying the single-particle states defined by Eqs. (22)-(24).

The thermodynamical potential $\Omega$ can be given explicitly in terms of the energy eigenvalues $E_{i}(\mathbf{p})$ of the quasinucleons and the parameters of the meson fields as follows:

$$
\begin{aligned}
& \Omega=\Omega_{\text {nucleon }}+\Omega_{\text {meson }} \\
& \Omega_{\text {nucleon }}=-T \int_{-\infty}^{\infty} d p_{1} \int_{-\infty}^{\infty} d p_{2} \int_{-k / 2}^{+k / 2} d p_{3} \sum_{i} \ln \left(1+e^{-\left[E_{i}(\mathbf{p})-\mu\right] / T}\right) \\
& \Omega_{\text {meson }}=V\left[\frac{1}{2} m_{\sigma}^{2} \bar{\sigma}^{2}+U(\bar{\sigma})+\frac{1}{2}\left(m_{\omega}^{2}+k^{2}\right) \bar{\omega}^{2}-\frac{1}{2} m_{\omega}^{2}\left(\bar{\omega}^{0}\right)^{2}\right]
\end{aligned}
$$

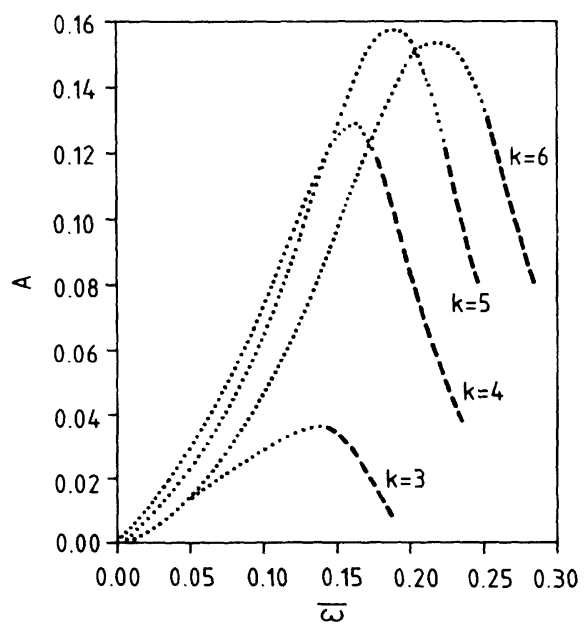

FIG. 3. Anisotropy $A$ defined in (38) as the function of the field amplitude $\bar{\omega}$ at various $k$ values. Dotted lines, unstable solutions; dashed lines, stable solutions.

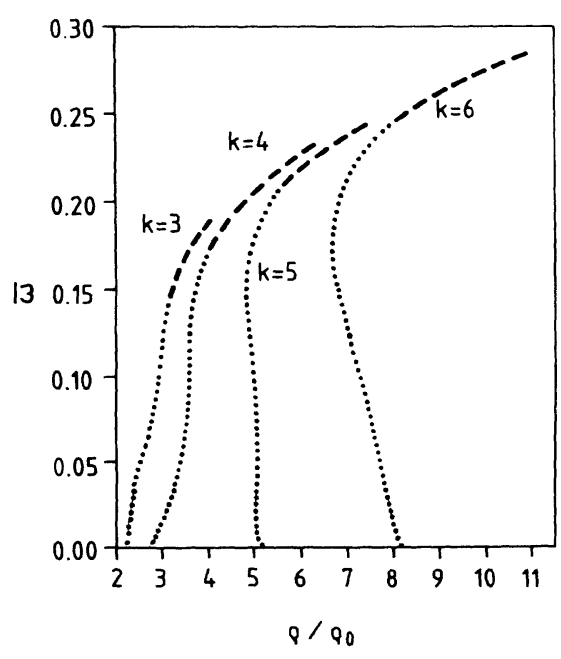

FIG. 4. Field amplitude $\bar{\omega}$ as the function of the nucleon density $\rho / \rho_{0}$ at various $k$ values. $\rho_{0}=0.193 \mathrm{fm}^{-3}$ is the density of normal nuclear matter. Dotted lines, unstable solutions; dashed lines, stable solutions. 


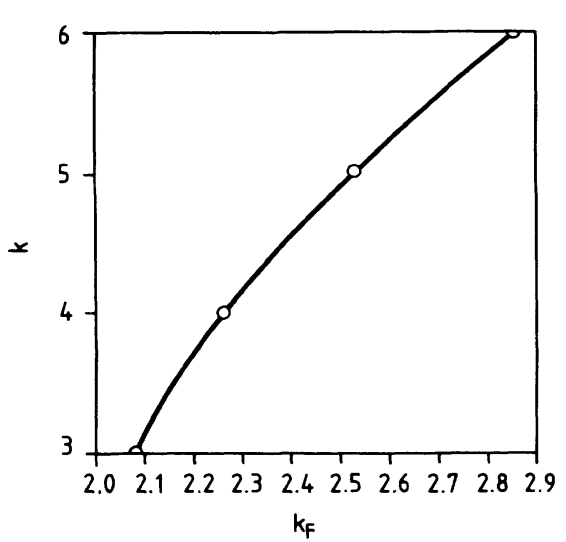

FIG. 5. Relation between the wave number $k$ and Fermi momentum $k_{F}$ at which stable periodic solutions appear.

where the volume of the system is denoted by $V$.

At a fixed value of $\mu, T$, and $V$, the necessary conditions of the thermodynamical equilibrium can be expressed by the following requirements:

$$
\frac{\partial \Omega}{\partial \bar{\sigma}}=0, \frac{\partial \Omega}{\partial \bar{\omega}^{0}}=0, \frac{\partial \Omega}{\partial \bar{\omega}}=0, \frac{\partial \Omega}{\partial k}=0 .
$$

From the numerical studies it turned out that these equations can be satisfied simultaneously only if

$$
\bar{\omega}=0 \text {. }
$$

This means that thermodynamical equilibrium can be obtained only if the $\omega$ field is constant in space and then the parameter $k$ is irrelevant. Let us drop the requirement

$$
\frac{\partial \Omega}{\partial k}=0 \text {. }
$$

Then the set of equations,

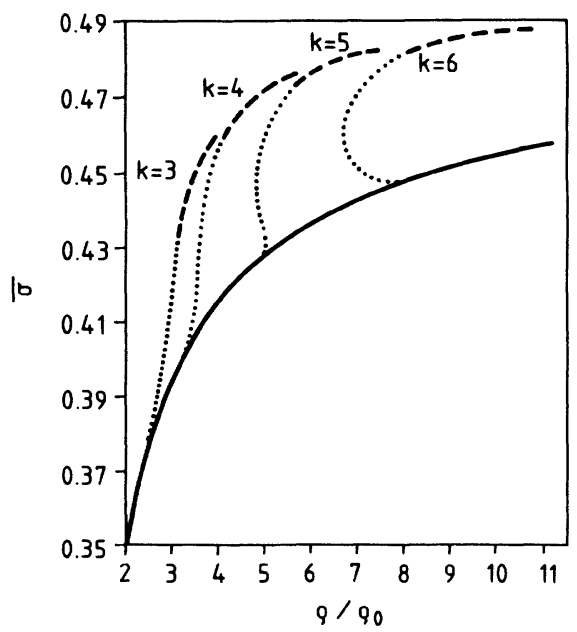

FIG. 6. Field amplitude $\bar{\sigma}$ plotted against nucleon density $\rho / \rho_{0}$. Solid line shows the normal phase solutions. Dotted and dashed lines show the periodic phase unstable and stable solutions, respectively.

$$
\frac{\partial \Omega}{\partial \bar{\sigma}}=0, \frac{\partial \Omega}{\partial \bar{\omega}^{0}}=0, \frac{\partial \Omega}{\partial \bar{\omega}}=0,
$$

does have a solution, which is identical with the solution of the self-consistent set of the equations given by Eqs. (11)-(16). This means that if the "wave number" $k$ of the $\omega$ field is considered as a parameter defined by the external conditions and it is fixed, then a self-consistent periodic $\omega$ field with a nonvanishing amplitude $\bar{\omega} \neq 0$ can be formed.

Let us investigate the stability of the self-consistent solution. For this purpose we calculate numerically the second derivatives of the thermodynamical potential. The solutions can be classified into two groups. In a number of cases,

$$
\frac{\partial^{2} \Omega}{\partial \bar{\sigma}^{2}}>0, \frac{\partial^{2} \Omega}{\partial \bar{\omega}^{2}}>0,
$$

and so there is a minimum in the thermodynamical potential; in other words, the equilibrium is stable. However, in other cases where

$$
\frac{\partial^{2} \Omega}{\partial \bar{\sigma}^{2}}>0 \text { but } \frac{\partial^{2} \Omega}{\partial \bar{\omega}^{2}}<0,
$$

which corresponds to a saddle point of $\Omega$, the equilibrium is not stable.

To clarify the situation, we calculate the anisotropy [14] of the system defined by the help of the pressure tensor components in the following form:

$$
A=\frac{P_{\|}-P_{\perp}}{P_{\|}+P_{\perp}},
$$

where the pressure parallel and perpendicular compared with $\mathbf{k}$ are denoted by $\boldsymbol{P}_{\|}$and $\boldsymbol{P}_{\perp}$, respectively.

\section{RESULTS}

In the framework of quantum hadrodynamics, a periodic $\omega$ field has been found which is associated in a self-consistent way with a periodic structure of the nucleon current while the density of the nuclear matter remains constant. This is in contrast with other periodic structures discussed in the literature up to now, where the periodic structure is also present in the density.

It was found that, in the presence of a self-consistent periodic $\omega$ field, the pressure is anisotropic. With increasing amplitude $\bar{\omega}$, the anisotropy $\boldsymbol{A}$ increases almost linearly up to a maximum, and afterward it decreases again almost linearly (Fig. 3).

The amplitude $\bar{\omega}$ is a double-valued function of the anisotropy $\boldsymbol{A}$ for a given $k$ value. Investigating the stability of the solutions, a stable minimum in $\Omega$ has been found on the decreasing branch of the $A(\bar{\omega})$ function, while the increasing branch corresponds to a saddle point in $\Omega$. (Dashed lines designate stable periodic solutions and dotted lines designate unstable ones in the figures.)

Looking at the field amplitude $\bar{\omega}$ as a function of the nucleon density, it is striking that the curve is very steeply rising as far as the point where stable solutions appear (Fig. 4). This means that the density and $k$ are strongly correlated. 

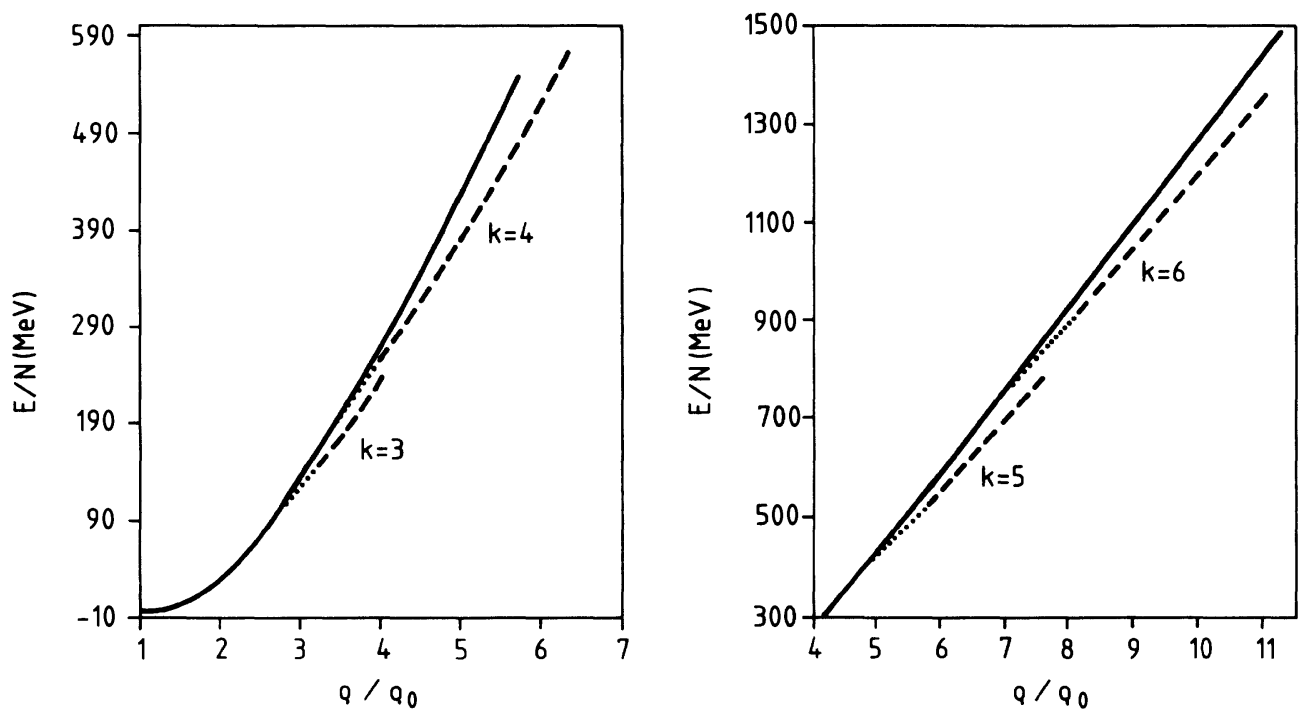

FIG. 7. Binding energy per nucleon $E / N$ plotted against nucleon density $\rho / \rho_{0}$ for various $k$ values. Solid lines show the normal phase solutions. Dotted and dashed lines show the periodic phase unstable and stable solutions, respectively.

Let us denote by $k_{F}$ the Fermi momentum defined as

$$
k_{F}=\left(\frac{3 \pi^{2}}{2} \rho\right)^{1 / 3} \text {. }
$$

One can see in Fig. 5 that the wave number $k$ of the $\omega$ field increases almost linearly with the $k_{F}$ values at which stable solutions appear.

Presumably, in a phase transition, the pressure anisotropy determines the field amplitude $\bar{\omega}$ and the nucleon density determines the "lattice spacing" $2 \pi / k$.

In Figs. 6-8 the periodic phase solutions are compared to the normal phase of nuclear matter where

$$
\begin{aligned}
& \sigma(x) \rightarrow\langle\sigma(x)\rangle=\bar{\sigma} \\
& \omega^{0}(x) \rightarrow\left\langle\omega^{0}(x)\right\rangle=\bar{\omega}^{0}
\end{aligned}
$$

are constant and $\omega^{1}=\omega^{2}=\omega^{3}=0$. (Solid lines correspond to the normal phase in these figures.)

For every prescribed value of $k(\neq 0)$, both the binding energy per nucleon, $E / N$, and the average pressure $P=\left(P_{\|}+2 P_{1}\right) / 3$ as a function of the nucleon density are smaller in the periodic phase.

It can be seen that, for higher $k$ values ( $k=5$ and 6 ), the field amplitude $\bar{\sigma}$ and average pressure are doublevalued functions of the nucleon density for the branch of the unstable solutions, but they are single valued in the intervals corresponding to stable ones.

It was found that the formation of the periodic structure depends on the softness of the nuclear equation of state. Using the parameters given by Walecka ( $b=0$ and $c=0$ ) [9], periodic self-consistent solutions are found above a critical density. Assuming a self-interacting $\sigma$

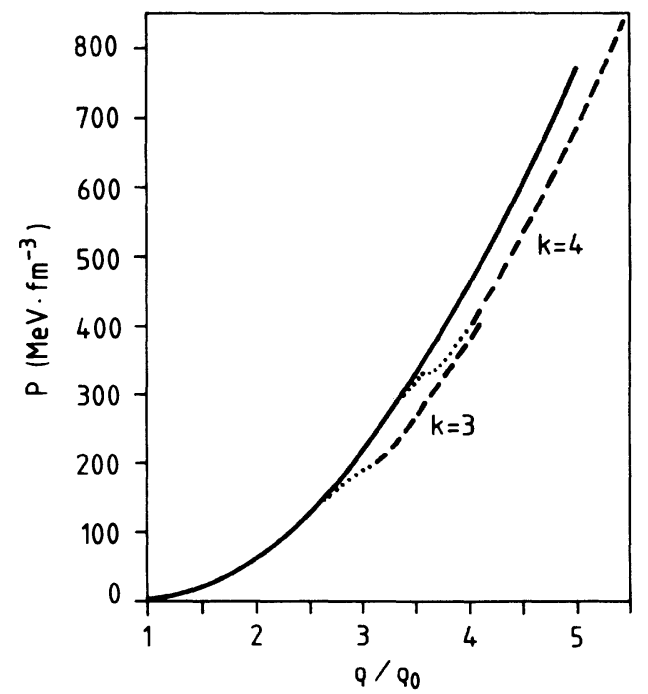

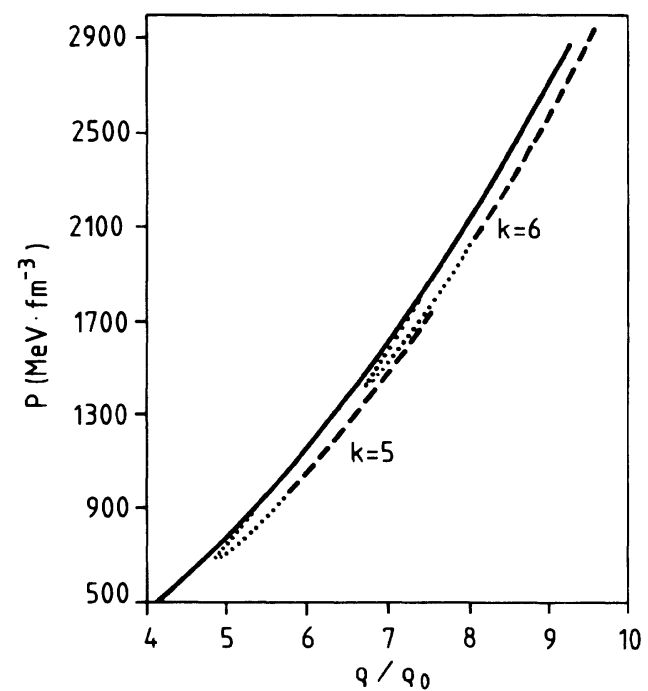

FIG. 8. Average pressure $P=\left(P_{\|}+2 P_{1}\right) / 3$ plotted against nucleon density $\rho / \rho_{0}$ for various $k$ values. Solid lines show the normal phase solutions. Dotted and dashed lines show the periodic phase unstable and stable solutions, respectively. 
field giving rise to a soft equation of state [15], we were unable to find any periodic self-consistent solution even at much higher densities. The results shown in the figures were calculated by using the Walecka parameters.

For the sake of simplicity of the presentation, we have not mentioned the role of the antinucleons. Their contribution, however, has been checked and found to be negligible at low temperatures (below $50 \mathrm{MeV}$ ). At higher temperatures the contribution of the antinucleons must be included.

\section{ACKNOWLEDGMENTS}

The calculations were carried out on the MicroVAX computer donated by the Alexander von Humboldt Foundation.

\section{APPENDIX}

To calculate the derivatives of the thermodynamical potential $\Omega$, the eigenfunctions are not needed. The energy eigenvalues can be obtained by solving Klein-Gordontype equations. The procedure is the following. Let us write the Dirac equation as

$$
\left(\gamma^{\mu} \pi_{\mu}-m^{*}\right) \psi(x)=0,
$$

where

$$
\pi_{\mu}=i \partial_{\mu}-g_{\omega} \omega_{\mu}
$$

and

$$
m^{*}=m-g_{\sigma} \bar{\sigma} .
$$

Multiplying by $\left(\gamma^{\mu} \pi_{\mu}+m^{*}\right)$, we get

$$
\left[\pi^{\mu} \pi_{\mu}-\left(m^{*}\right)^{2}+\frac{1}{2} g_{\omega} \sigma^{\mu v} F_{\mu v}\right] \psi=0,
$$

where $F_{\mu v}$ is the field tensor defined in (18) and

$$
\sigma^{\mu v}=\frac{i}{2}\left[\gamma^{\mu}, \gamma^{v}\right]
$$

Writing $\psi$ as given in $(22)-(24)$ and considering the Lorentz condition (17), as well as

$$
\begin{aligned}
& k_{\mu} \omega^{\mu}=0, \\
& k_{\mu} k^{\mu}=-k^{2},
\end{aligned}
$$

we get

$$
\begin{array}{r}
k^{2} \Phi_{i}^{\prime \prime}(\xi)-2 i k p^{3} \Phi_{i}^{\prime}(\xi)+\left[p_{\mu} p^{\mu}-2 g_{\omega} p_{\mu} \omega^{\mu}+g_{\omega}^{2} \omega_{\mu} \omega^{\mu}\right. \\
\left.-\left(m^{*}\right)^{2}+\frac{1}{2} g_{\omega} \sigma^{\mu \nu} F_{\mu \nu}\right] \Phi_{i}=0
\end{array}
$$

where

$$
\xi=k z \text {. }
$$

Taking into account the Ansätze given by (6)-(9), the following set of equations can be obtained:

$$
\sum_{n^{\prime}} A_{n n^{\prime}} u_{i}\left(\mathrm{p}, n^{\prime}\right)=K_{i} u_{i}(\mathrm{p}, n)
$$

where the matrix to be diagonalized is given as

$$
\begin{aligned}
A_{n n^{\prime}}= & \left(k^{2} n^{2}-2 p^{3} k n\right) \delta_{n n^{\prime}}-S^{(-)} \delta_{n^{\prime}, n+1} \\
& -S^{(+)} \delta_{n^{\prime}, n-1} \\
S^{( \pm)}= & \frac{g_{\omega} \bar{\omega}}{2}\left[2\left(p^{1} \pm i p^{2}\right)-k\left(\sigma^{1} \pm i \sigma^{2}\right)\right] \\
\sigma^{1} \equiv & \sigma^{23}=i \gamma^{2} \gamma^{3} \\
\sigma^{2} \equiv & \sigma^{31}=i \gamma^{3} \gamma^{1} .
\end{aligned}
$$

The eigenvalue $K_{i}$ has the following form:

$K_{i}=p_{0 i}^{2}-\mathbf{p}^{2}-2 g_{\omega} p_{0 i} \bar{\omega}_{0}+g_{\omega}^{2}\left(\bar{\omega}_{0}^{2}-\bar{\omega}^{2}\right)-\left(m^{*}\right)^{2}$

From here, the expression for the energy eigenvalue $E_{i}$ can be obtained as follows:

$E_{i} \equiv p_{0 i}=g_{\omega} \bar{\omega}_{0} \pm\left[K_{i}+\mathbf{p}^{2}+\left(m^{*}\right)^{2}+g_{\omega}^{2} \bar{\omega}^{2}\right]^{1 / 2}$.
[1] G. Nicolis and I. Prigogine, Self-organization in Nonequilibrium Systems (Wiley, New York, 1977); H. Haken, Synergetics (Springer, Berlin, 1977).

[2] A. B. Migdal, Zh. Eksp. Teor. Fiz. 61, 2209 (1971); V. Ruck, M. Gyulassy, and W. Greiner, Z. Phys. A 277, 391 (1976); M. Gyulassy and W. Greiner, Ann. Phys. (N.Y.) 109, 485 (1977).

[3] Yu. B. Ivanov, Nucl. Phys. A474, 669 (1987); A474, 693 (1987).

[4] B. L. Friman and P. Henning, Phys. Lett. B 206, 579 (1988); P. Henning and B. L. Friman, Nucl. Phys. A490, 689 (1989).

[5] K. Lim and C. J. Horowitz, Nucl. Phys. A501, 729 (1989); R. J. Furnstahl and C. J. Horowitz, ibid. A485, 632 (1988).

[6] C. E. Price, J. R. Shepard, and J. A. McNeil, Phys. Rev. C 41, 1234 (1990); 42, 247 (1990).

[7] A. W. Overhauser, Phys. Rev. Lett. 4, 415 (1960); Phys. Rev. 128, 1437 (1962).

[8] M. Noga, Nucl. Phys. B220, 185 (1982).
[9] J. D. Walecka, Ann. Phys. (N.Y.) 83, 491 (1974); B. D. Serot and J. D. Walecka, The Relativistic Nuclear ManyBody Problem, in Advances in Nuclear Physics, edited by $\mathrm{J}$. W. Negele and E. Vogt (Plenum, New York, 1986), Vol. 16.

[10] R. J. Furnstahl, R. J. Perry, and B. D. Serot, Phys. Rev. C 40, 321 (1989).

[11] S. A. Chin, Ann. Phys. (N.Y.) 108, 301 (1977).

[12] J. Frank, in Proceedings of the International Workshop on Gross Properties of Nuclei and Nuclear Excitations XVII, Hirschegg, Austria, 1989, edited by H. Feldmeier (GSI Darmstadt, West Germany, 1989).

[13] I. Lovas, J. Németh, and K. Sailer, Nucl. Phys. A430, 731 (1984).

[14] I. Lovas, Nucl. Phys. A367, 509 (1981).

[15] J. Boguta and A. R. Bodmer, Nucl. Phys. A292, 413 (1977); I. Lovas and Gy. Wolf, Acta Phys. Hung. 58, 23 (1985). 